\title{
Single Nucleotide Polymorphisms of Indoleamine 2,3-Dioxygenase 1 Influenced the Age Onset of Parkinson's Disease
}

Nóra Török ${ }^{1,5}$, Rita Maszlag-Török ${ }^{1}$, Kinga Molnár1 ${ }^{1}$, Zoltán Szolnoki², Ferenc Somogyvári³, Krisztina Boda ${ }^{4}$, Masaru Tanaka ${ }^{1,5}$, Péter Klivényi ${ }^{1}$, László Vécsei ${ }^{1,5, *}$

${ }^{1}$ Department of Neurology, Faculty of Medicine, Albert Szent-Györgyi Clinical Centre, University of Szeged, 6 Semmelweis u., H-6725 Szeged, Hungary

${ }^{2}$ Department of Neurology and Cerebrovascular Diseases, Pándy Kálmán County Hospital, 1 Semmelweis u., H-5700 Gyula, Hungary

${ }^{3}$ Department of Medical Microbiology and Immunobiology, University of Szeged, 10 Dóm tér, H-6725 Szeged, Hungary

${ }^{4}$ Department of Medical Physics and Informatics, University of Szeged, 9 Korányi fasor, H-6720 Szeged, Hungary

${ }^{5}$ MTA-SZTE Neuroscience Research Group, 6 Semmelweis u., H-6725 Szeged, Hungary

Correspondence: László Vécsei, vecsei.laszlo@med.u-szeged.hu; Tel: +36(62)545 351, 545348

\section{Highlights}

- Disturbance of the tryptophan-kynurenine pathway is observed in Parkinson's disease (PD).

- The single nucleotide polymorphisms (SNP) of the indoleamine 2,3-dioxygenase (IDO) are present.

- No significant correlation was found in rs34155785, rs7820268, and rs9657182 SNPs between PD patients and healthy controls.

- A alleles of rs7820268 SNP or rs9657182 SNP carriers are prone to the later onset of PD than non-carriers.

\begin{abstract}
Aims

Earlier studies reported alterations of the kynurenine (KYN) pathway of tryptophan (TRP) metabolism in Parkinson's disease (PD). The first rate-limiting enzymes indoleamine 2,3dioxygenase (IDO) and tryptophan dioxygenase were observed upregulated, resulting elevated KYN/TRP ratios in the serum and cerebrospinal fluid samples of patients with PD. An increasing number of single nucleotide polymorphisms (SNPs) has been identified in a population of PD. However, little is known if genetic variations of the IDO contribute to disturbance of the KYN metabolism in and the pathogenesis of PD.

Main methods

SNP analysis of IDO1 was performed by allelic discrimination assay with fluorescently labelled TaqMan probes and a subgroup analysis was conducted according to the age of PD onset. The frame shifts variant rs34155785, intronic variant rs7820268, and promotor region variant rs9657182 SNPs of 105 PD patients without comorbidity were analyzed and compared to 129 healthy controls.
\end{abstract}

Key findings

No significant correlation was found in three SNPs between PD patients and healthy controls. However, the subgroup analysis revealed that A alleles of rs7820268 SNP or rs9657182 SNP carriers contribute to later onset of PD than non-carriers.

Significance

The study suggested that SNPs of IDO1 influenced the age onset of PD and genotyping of SNPs in certain alleles potentially serves as a risk biomarker of PD. 


\section{Keywords}

Kynurenines; Tryptophan; Indoleamine 2,3-dioxygenase; Single nucleotide polymorphisms; Parkinson's diseases; Neurodegenerative diseases

\section{Introduction}

Parkinson's disease (PD) is the second most prevalent chronic progressive neurodegenerative disease characterized by motor symptoms such as tremor, rigidity, and hypokinesia. Relatively significant symptoms are autonomic dysfunction including arrythmia, blood pressure irregularity, asymmetric sweating, and incontinence and psychobehavioral manifestations including dementia, depression, anxiety, paranoia, and psychosis. The non-motor symptoms may appear in early stages of the disease and even before the appearance of classical motor symptoms [1-3]. The histopathological and clinical hallmarks of PD include the degeneration of dopaminergic neurons in the substantia nigra pars compacta, the presence of Lewy bodies, and the positive response to dopamine (DA) replacement therapy. The disease affects primarily the elderly, imposing a serious burden on the aging societies. The prevalence of PD is approximately $0.2 \%$ on average in the general population, but it is increasing with age up to $1.9 \%$ [4].

The etiology of PD remains poorly understood. Besides genetic disposition, pathological findings are abnormal protein aggregation, elevated oxidative stress, mitochondrial dysfunction, increased glutamate excitotoxicity, alteration of immune response, disturbance of kynurenine pathway (KP) of tryptophan (TRP) metabolism, and among others [5-10]. Altered levels of and ratios of kynurenine $(\mathrm{KYN})$ metabolites have been observed in neurologic and psychiatric diseases [3,1116]. Earlier studies revealed that the activities of indoleamine 2,3-dioxygenase (IDO) 1/TRP 2,3dioxygenase (TDO) were upregulated in PD patients compared to controls, which was indicated by elevation of L-KYN/TRP ratios in the serum and in cerebrospinal fluid (CSF) samples of the patients [17]. The cascade of KP produces several neuroactive metabolites such as neurotoxic 3hydroxykynurenine (3-HK) quinolinic acid (QUIN), 3-hydroxyanthranillic acid (3-HAA) and neuroprotective kynurenic acid (KYNA).

3-HK and 3-HAA generate reactive oxygen species (ROS) [10]. An elevation of 3-HK levels was related to excitotoxic injury and is observed in patients with neurodegenerative diseases [3]. The neurotoxic effects of 3-HK and 3-HAA involve the generation of superoxide anion and hydrogen peroxide, which contribute to the oxidative processes implicated in the pathophysiology of meningitis [18]. QUIN is a free-radical metabolite. Interferon (IFN)- $\gamma$ activates IDO, formamidase, and kynurenine 3-monooxygenase (KMO) activities in human microglial cells and macrophages, increasing QUIN synthesis [19].

KYNA is a broad-spectrum, competitive antagonist of all three ionotropic excitatory glutamate receptors including $\alpha$-amino-3-hydroxy-5-methyl-4-isoxazolepropionic acid (AMPA) receptor, kainate receptor, and $N$-methyl-D-aspartate (NMDA) receptor [20,21]. However, KYNA exhibited dual actions at AMPA receptors dependent on its concentration: inhibitory at micromolar concentrations, while excitatory at nanomolar concentrations. The micromolar concentrations are inhibitory, while the nanomolar concentrations are facilitatory by allosteric modulation of the AMPA receptor [22.23]. The actions of KYNA at the $\alpha-7$ nicotinic acetylcholine receptor remain controversial [24]. KYNA has antioxidant properties that scavenges ROS to suppress overshooting inflammation in damaging tissues. Reduced concentrations of KYNA may contribute to tissue damage and inflammatory cell proliferation neurodegenerative diseases $[3,10,25,26]$. In addition, KYNA was reported to contribute to weight reduction without affecting osteogenesis [27].

The indoleamine 2,3-dioxygenase IDO1 is one of the first rate-limiting enzymes that converts L-TRP to N-formyl KYN in TRP metabolism, which play a crucial role in governing concentrations of downward bioactive KYN metabolites (Figure 1) [10]. Activation of IDO1 and the KYN system promotes immunosuppressive effects by inhibition of Natural Killer cells, inhibition of $\mathrm{T}$ cell functions, and activation of the regulatory $\mathrm{T}$ cells [28]. Firstly, single nucleotide polymorphisms (SNPs) analysis was performed in three loci of IDO1 in PD patients and healthy controls. The 
frameshift mutation rs34155785 SNP causes a drastic change in the gene product. Both intronic variant rs7820268 SNP, and promotor region variant rs9657182 SNP affect the immune system. The $\mathrm{T}$ allelic variants of the rs7820268 showed impaired $\mathrm{CD} 8^{+}$regulatory $\mathrm{T}$ cell function, while the rs9657182 SNP was found more susceptible to IFN-alpha treatment, which induced depressive symptoms [29,30] (Figure 2). Secondly, a subgroup analysis was conducted according to the age of PD onset in search of the genetic link between the IDO1 variants and PD.

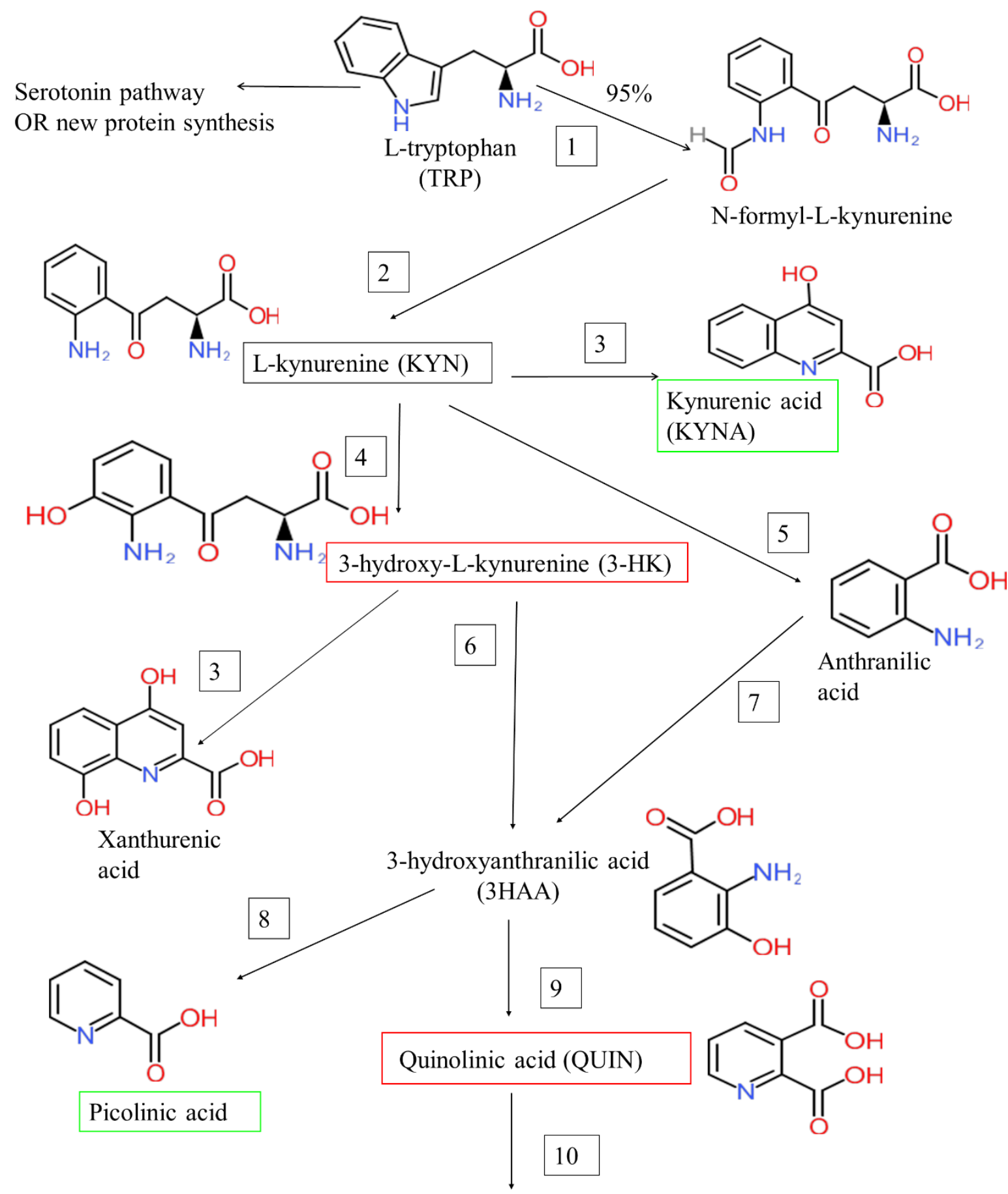

Nicotinamide adenosine dinucleotide (NAD)

Figure 1. The kynurenine pathway (KP) is the degradation route of tryptophan (TRP) metabolism producing an end-product nicotinamide adenine dinucleotide (NAD). The indoleamine 2,3-dioxygenase (IDO) 1, IDO2, and the tryptophan 2,3-dioxygenase (TDO) (1) are the first rate-liming enzymes that convert the Ltryptophan (TRP) to $N$-formyl-L-kynurenine. $N$-formyl-L-kynurenine is converted by formamidase (2) to L- 
kynurenine (L-KYN) (black bracket). L-KYN is metabolized into various bioactive compounds: the neuroprotective metabolites are kynurenic acid and picolinic acid (green brackets), while the neurotoxic ones are the 3-hydroxy-L-kynurenine (3-HK) and the quinolinic acid (red brackets). The following are main enzymes of the KP: 1: tryptophan dioxygenase (TDO) and indoleamine 2,3-dioxygenase (IDO), 2: formamidase, 3 : kynurenine aminotransferases (KATs), 4: kynurenine 3-monooxygenase (KMO), 5: kynureninase, 6 : kynureninase, 7: non-specific hydroxylation, 8: 2-amino-3-carboxymuconate-semialdehyde decarboxylase, 9: 3hydroxyanthranilic acid oxygenase, 10: quinolinic acid phosphoribosyltransferase

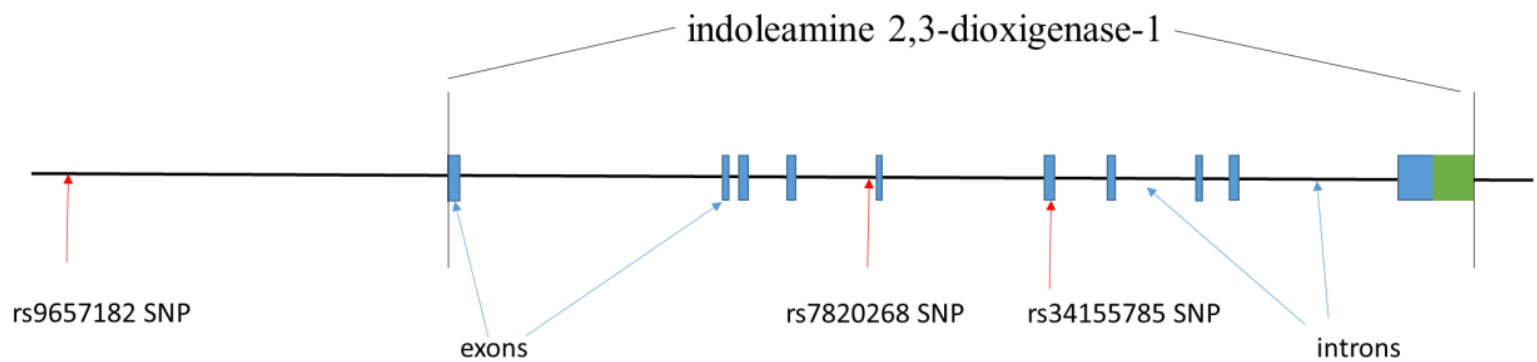

Figure 2. Schematic of the indoleamine 2,3-dioxygenase (IDO) 1 gene. The IDO1 gene consist of 10 exons and 9 introns localized in 8p11.21 chromosome region. It has 9 transcript variants. The encoded protein is 403 amino acid long. The rs9657182 SNP is located 5' upstream from the promoter region of the IDO1 gene. The rs7820268 SNP is localized in the fourth intron and the last rs34155785 SNP, which is a frame shift mutation, is located in the sixth exon.

\section{Materials and Methods}

\subsection{Ethics}

The blood samples of 105 PD patients and 129 healthy controls were examined for the study. All patients gave their informed consent in accordance with the Declaration of Helsinki, and the study was approved by the Medical Research Council Scientific and Research Ethics Committee (470663/2013/EKU (556/2013)). The samples were collected at two sites (at the Department of Neurology, Faculty of Medicine, University of Szeged, Hungary, and at the Department of Neurology and Cerebrovascular Diseases, Pándy Kálmán County Hospital, Gyula, Hungary). The patient and the control groups were age- and gender-matched (gender ratio $p=0.989$; mean age, $p=0.310$ ). The onset of the disease could not be established for two patients. The sociodemographic data of the two groups are presented in Table 1.

Table 1. the sociodemographic data of PD patients and controls.

\begin{tabular}{|l|r|r|r|r|r|r|r|}
\hline Group & \multicolumn{1}{|l|}{ Male } & Female & Mean age & Median & \multicolumn{1}{l|}{ Min } & \multicolumn{1}{l|}{ Max } & Age at onset \\
\hline $\begin{array}{l}\text { PD } \\
\text { patients } \\
(105)\end{array}$ & 48 & 57 & $66.42 \pm 9.24$ & 68 & 34 & 84 & $58.81 \pm 10.970$ \\
\hline $\begin{array}{l}\text { Controls } \\
(129)\end{array}$ & 58 & 71 & $65.26 \pm 8.1$ & 63 & 53 & 87 & - \\
\hline
\end{tabular}

Min: minimum age in the group, Max: maximum age in the group

\subsection{Methods}

\subsubsection{DNA isolation and polymerase chain reaction}

Peripheral whole blood samples (stored at $-80^{\circ} \mathrm{C}$ ) were subjected to genomic DNA isolation by the desalting method developed by Miller et al [57]. The purified genomic DNA were stored at $-20^{\circ} \mathrm{C}$ at the 
biobank of the Department of Neurology, Faculty of Medicine, University of Szeged (biobank license: Regional Human Biomedical Research Ethics Committee: 135/2008).

The IDO1 genotypes were determined by allelic discrimination study with TaqMan probes. Three SNP of the IDO1 gene were investigated. The rs34155785SNP is a frame shift mutation in the human genome (results a Phe (F)>Leu (L) exchange), which localize in the sixth exon of the IDO1 gene. The rs7820268SNP is intronic SNP (G/A change) of the IDO1 gene is localized in the fourth intron. The rs9657182SNP (G/A change) is localized 5' upstream from the beginning of the IDO1 promoter region.

For the rs $34155785 \mathrm{SNP}$, the following primers were used for the amplification of the DNA forward primer: 5'- CTA AAC TTC TTG CCT TCC TTA TC-3'; reverse primer: 5'- AGA CGT ACT TTG ATT GCA GA-3'. The following probes were applied for allelic discrimination: wild type allele: $5^{\prime}$-Fam- GAC GTT TTG TTC TCA TTT CGT G-BHQ-1-3'; and C allele: 5'-Hex- GAC GTT TTG TTG CTC ATT TCG TG-BHQ-1-3'.

The rs7820268 SNP, the following primers were applied for the amplification of the G/A at chromosome 8: forward primer: 5' - TAA ATG TAA TGC CTA CTG AAG AA-3'; reverse primer: 5'CCT TAT GAA AGC AGC CAT G- $3^{\prime}$. The following probes were designed for allelic discrimination: $G$ allele: 5'-Fam- GTA GCA TTC AAT CAA ATA GCA ACA AC-1-3'; and A allele: 5'-Hex- GTA GCA TTC AAT TAA ATA GCA ACA AC-1-3'.

The rs9657182 SNP, the following primers were used for the: forward primer: $5^{\prime}$ - ATT GTT GTA GGT CAT AAA AGG AG-3'; reverse primer: 5'- TGA AGA CAC AAC ACT TAA GGA-3'. The following probes were applied for the separation of the alleles: $G$ allele: $5^{\prime}$-Fam- CCA TCT TTA ACC ACG GCC A-BHQ-1-3'; A allele: 5'-Hex- CCA TCT TTA ACC ATG GCC A-1-3'.

The parameters for PCR amplifications were as follows: $95^{\circ} \mathrm{C}$ for $3 \mathrm{~min}$, followed by 44 cycles of $95^{\circ} \mathrm{C}$ for $10 \mathrm{~s}$, and then $59^{\circ} \mathrm{C}$ for $50 \mathrm{~s}(\mathrm{rs} 34155785 \mathrm{SNP})$ or $57^{\circ} \mathrm{C}$ for $50 \mathrm{~s}\left(\mathrm{rs} 7820268 \mathrm{SNP}\right.$ ) or $55^{\circ} \mathrm{C}$ for $50 \mathrm{~s}$ (rs9657182 SNP). The genotyping specific master mix from the PCR Biosystem (2x PCRBio Genotyping mix Lo-ROX) was used. The PCR experiments were performed with a BioRad CFX96 C1000 real-time thermal cycler machine, and the data analysis was carried out with BioRad software (BioRad CFX Manager version 1.6).

\subsection{Statistical methods}

SPSS software version 26.0 was utilized for data analysis. We applied the chi-square test for comparing the distributions of the examined genotypes and alleles, and the t-test for comparing the averages in the two groups. The observed genotype frequencies of the PD and the control groups were in accordance with the Hardy-Weinberg equilibrium.

\section{Results}

The genotype distribution of rs34155785 was 105 (100\%) homozygous wild-types in the PD patient group, and $129(100 \%)$ in the control group, we did not detect the insertion allele in either group. The allele frequencies were $210(100 \%)$ wild-type alleles in the patient and $258(100 \%)$ in the control group (Table 2). This SNP variant is probably not associated with PD and do not affect the age at disease onset either, as no allelic differences could be observed between the two groups (Table 3) 
Table 2. Genotype and allele distribution of the investigated SNPs.

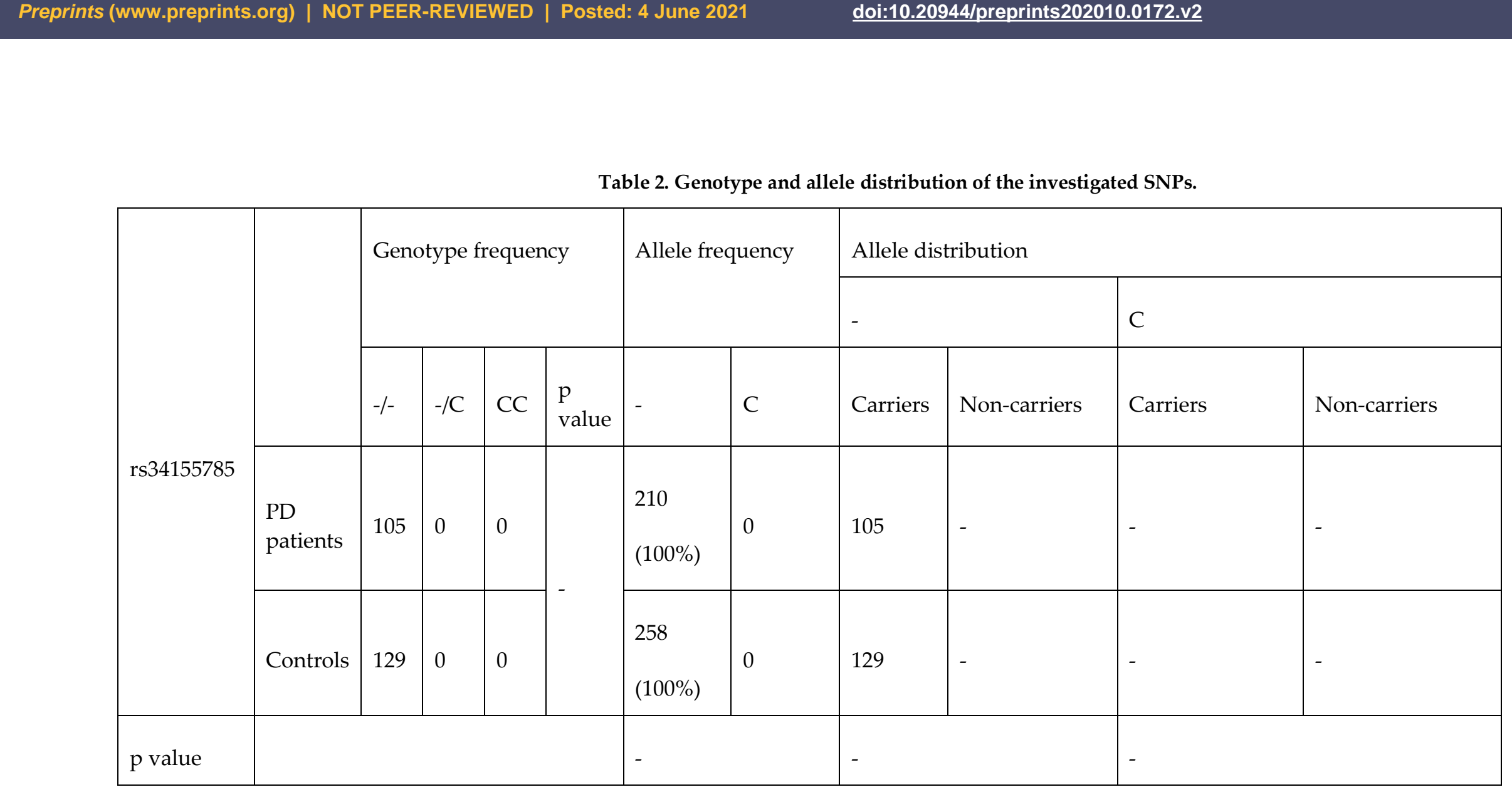


2 of 16

\begin{tabular}{|c|c|c|c|c|c|c|c|c|c|c|c|}
\hline & & & & & & \multicolumn{6}{|c|}{2 of 16} \\
\hline & & \multirow{2}{*}{\multicolumn{4}{|c|}{ Genotype frequency }} & \multirow{2}{*}{\multicolumn{2}{|c|}{ Allele frequency }} & \multicolumn{4}{|c|}{ Allele distribution } \\
\hline & & & & & & & & G & & $\mathrm{A}$ & \\
\hline \multirow{3}{*}{ rs7820268 } & & GG & AG & AA & $\mathrm{p}$ value & G & $\mathrm{A}$ & Carriers & Non-carriers & Carriers & Non-carriers \\
\hline & $\begin{array}{l}\text { PD } \\
\text { patients }\end{array}$ & 56 & 38 & 11 & \multirow{2}{*}{$\mathrm{p}=0.093$} & $\begin{array}{l}150 \\
(71.4 \%)\end{array}$ & $\begin{array}{l}60 \\
(28.6 \%)\end{array}$ & 94 & 11 & 49 & 56 \\
\hline & Controls & 59 & 63 & 7 & & $\begin{array}{l}181 \\
(70.2 \%)\end{array}$ & $\begin{array}{l}77 \\
(29.8 \%)\end{array}$ & 122 & 7 & 70 & 59 \\
\hline$p$ value & & & & & & $\mathrm{p}=0.763$ & & $\mathrm{p}=0.149$ & & $\mathrm{p}=0.248$ & \\
\hline
\end{tabular}


3 of 16

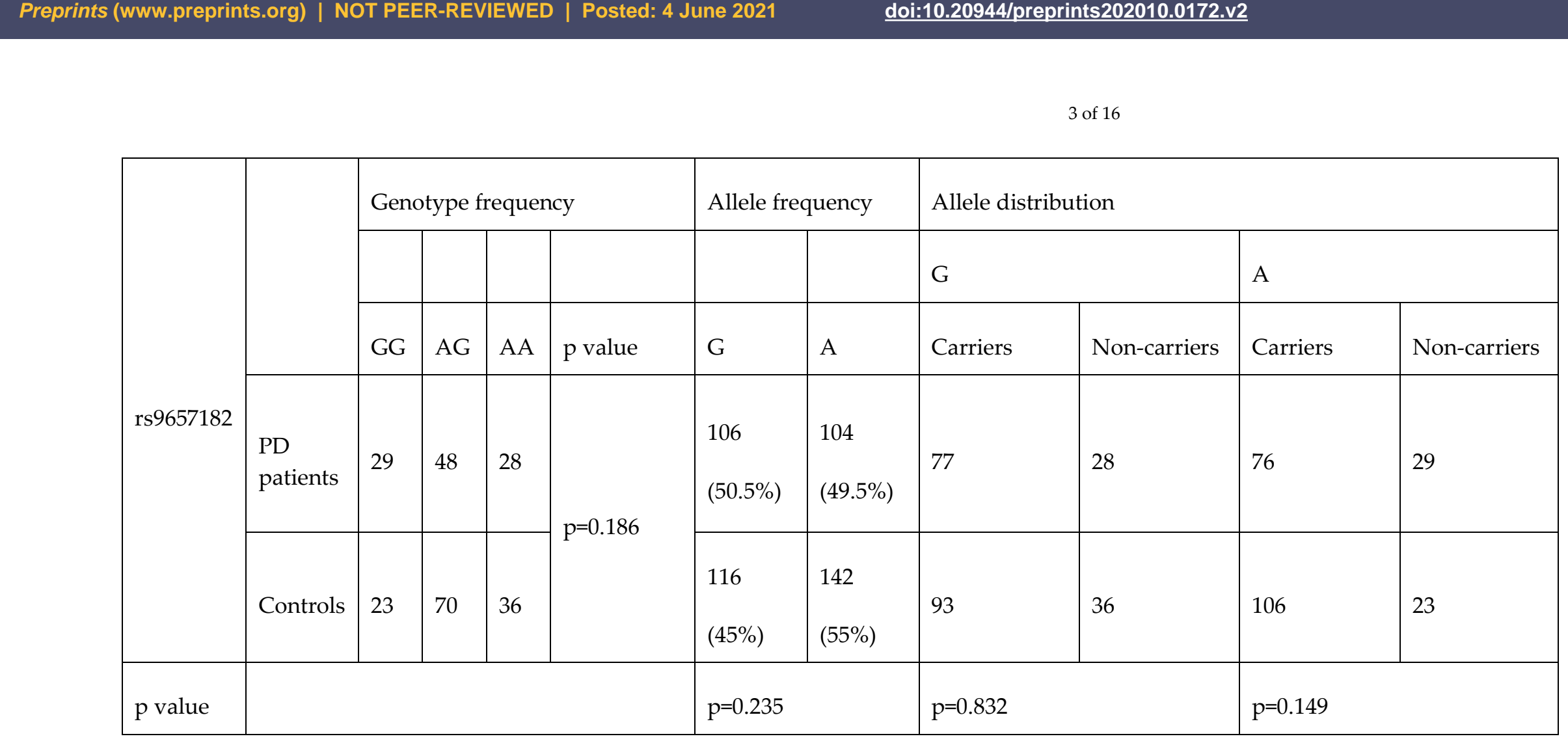


Table 2. Genotype frequency, allele distribution and age at onset of the PD patients.

\begin{tabular}{|c|c|c|c|c|}
\hline \multirow{2}{*}{\multicolumn{2}{|c|}{$\begin{array}{l}\text { Genotype frequency } \\
\text { rs7820268 }\end{array}$}} & \multicolumn{3}{|l|}{ Age at onset } \\
\hline & & Early onset & Late onset & $\mathrm{p}$ value \\
\hline \multicolumn{2}{|c|}{ GG } & $34(64.2 \%)$ & $21(42 \%)$ & \multirow[t]{3}{*}{$\mathrm{p}=0.042$} \\
\hline \multicolumn{2}{|c|}{ AG } & $13(24.5 \%)$ & $24(48 \%)$ & \\
\hline \multicolumn{2}{|c|}{ AA } & $6(11.3 \%)$ & $5(10 \%)$ & \\
\hline \multirow{2}{*}{\multicolumn{2}{|c|}{$\begin{array}{l}\text { Allele distribution } \\
\text { rs7820268 }\end{array}$}} & \multicolumn{3}{|l|}{ Age at onset } \\
\hline & & Early onset & Late onset & $\mathrm{p}$ value \\
\hline \multirow[t]{2}{*}{ G } & Carriers & $47(50.1 \%)$ & $45(48.9 \%)$ & \multirow[t]{2}{*}{$p=0.828$} \\
\hline & Non-carriers & $6(54.5 \%)$ & $5(45.5 \%)$ & \\
\hline \multirow[t]{2}{*}{$\mathrm{A}$} & Carriers & $19(39.6 \%)$ & $29(60.4 \%)$ & \multirow[t]{2}{*}{$\mathrm{p}=0.024$} \\
\hline & Non-carriers & $34(61.8 \%)$ & $21(38.2 \%)$ & \\
\hline \multirow{2}{*}{\multicolumn{2}{|c|}{$\begin{array}{l}\text { Genotype frequency } \\
\text { rs } 9657182\end{array}$}} & \multicolumn{3}{|l|}{ Age at onset } \\
\hline & & Early onset & Late onset & $\mathrm{p}$ value \\
\hline \multicolumn{2}{|c|}{ GG } & 19 (35.9\%) & $9(18 \%)$ & \multirow[t]{3}{*}{$p=0.091$} \\
\hline \multicolumn{2}{|c|}{ AG } & $23(43.4 \%)$ & $24(48 \%)$ & \\
\hline \multicolumn{2}{|c|}{ AA } & $11(20.8 \%)$ & 17 (34\%) & \\
\hline \multirow{2}{*}{\multicolumn{2}{|c|}{$\begin{array}{l}\text { Allele distribution } \\
\text { rs9657182 }\end{array}$}} & \multicolumn{3}{|l|}{ Age at onset } \\
\hline & & Early onset & Late onset & $\mathrm{p}$ value \\
\hline \multirow[t]{2}{*}{ G } & Carriers & 42 (56\%) & $33(44 \%)$ & \multirow[t]{2}{*}{$\mathrm{p}=0.131$} \\
\hline & Non-carriers & $11(39.3 \%$ & $17(60.7 \%)$ & \\
\hline $\mathrm{A}$ & Carriers & $34(45.3 \%)$ & $41(54.7 \%)$ & $\mathrm{p}=0.042$ \\
\hline
\end{tabular}




\begin{tabular}{|l|l|l|l|l|}
\hline & Non-carriers & $19(67.9 \%)$ & $9(32.1 \%)$ & \\
\hline
\end{tabular}

Early onset $<60$ years; Late onset $\geq 60$

The genotype distribution of rs7820268 in the patient cohort was 56 GG, 38 GA and 11 AA, and in the control group was $59 \mathrm{GG}, 63 \mathrm{GA}$ and $7 \mathrm{AA}$. The allele frequencies were very similar, with $71.4 \%$ $\mathrm{G}$ allele and 28.6\% A allele in the PD group and 70.2\% G and 29.8\% A in the controls (Table 2). This $\mathrm{SNP}$ variant is not significantly associated with the PD (genotype: $\mathrm{p}=0.093$, G allele: $\mathrm{p}=0.149, \mathrm{~A}$ allele: $p=0.248$ ), but it affects the age at disease onset (genotype: $p=0.042$, $G$ allele: $p=0.828$, A allele $\mathrm{p}=0.024$ ) (Table 3) (Figure 2A).

The impact of rs7820268 A allel on the age at onset

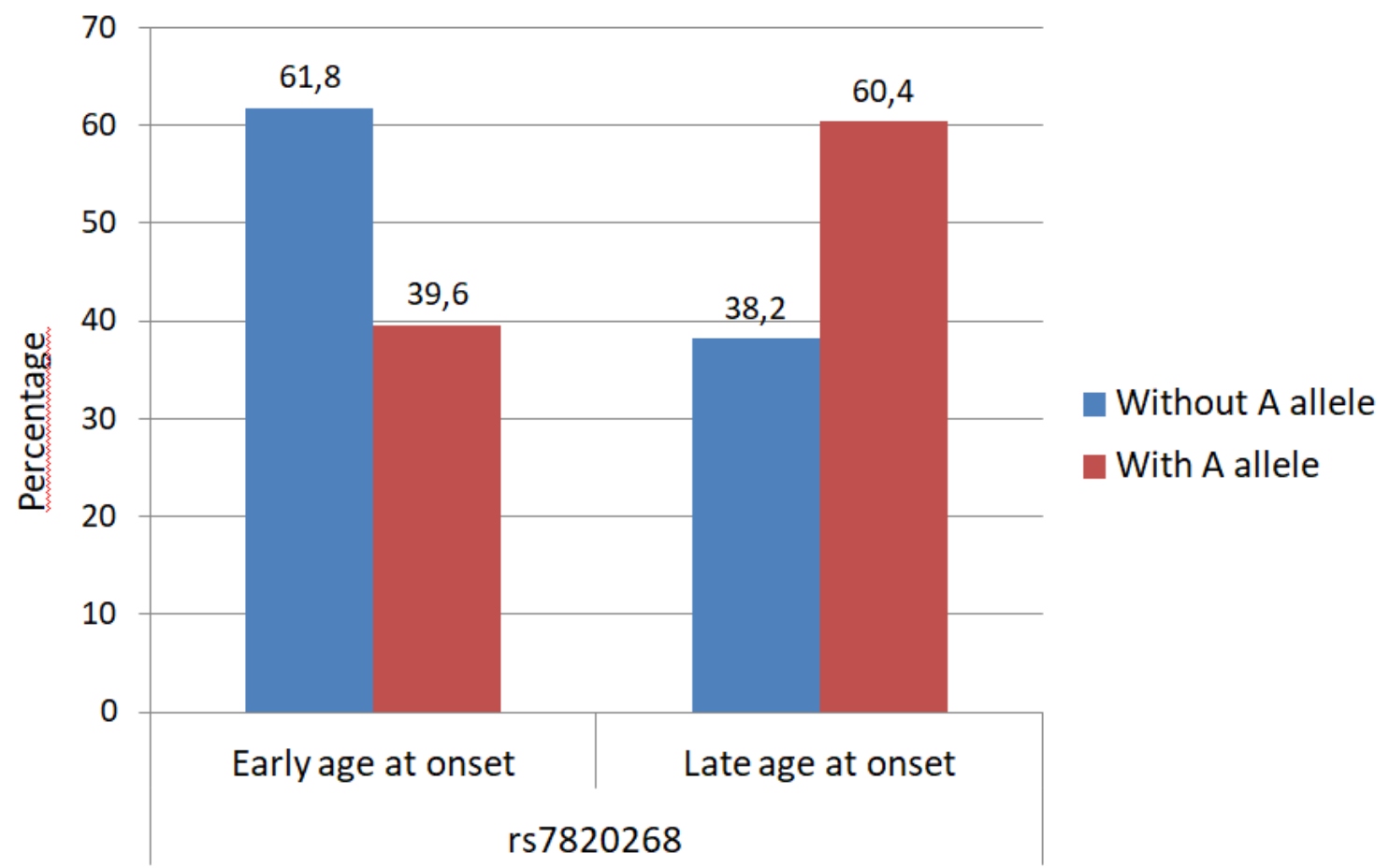

Figure 2A. The impact of the A allel of the IDO1 rs7820268SNP on the age at onset of PD. 
The impact of rs7820268 A allel on the age at onset

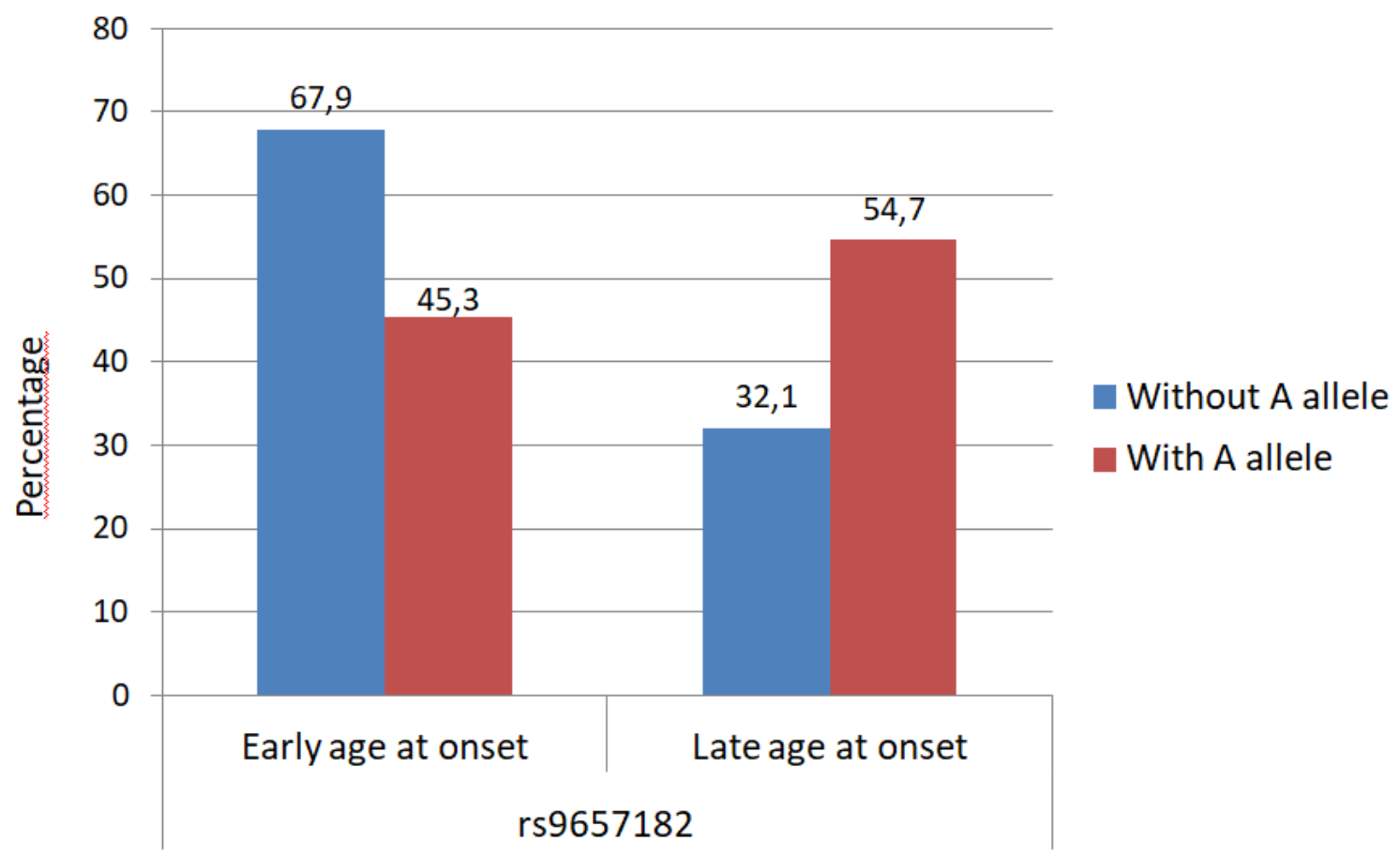

Figure 2B. The impact of the A allel of the IDO1 rs9657182SNP on the age at onset of PD.

The genotype distribution rs9657182 was 29 GG, 48 GA and 28 AA in the PD group, and 23 GG, $70 \mathrm{GA}$ and $36 \mathrm{AA}$ in the control group. The allele frequency results did not show remarkable differences, with $50.5 \% \mathrm{G}$ allele in the PD group vs $45 \% \mathrm{G}$ allele in the controls, and $49.5 \%$ A allele in the PD group vs 55\% A allele in the control group (Table 2). This SNP variant is likewise not associated with PD (genotype: $\mathrm{p}=0.186$, $\mathrm{G}$ allele: $\mathrm{p}=0.832$, A allele: $\mathrm{p}=0.073$ ), but it affects the age at disease onset (genotype: $\mathrm{p}=0.091$, $\mathrm{G}$ allele: $\mathrm{p}=0.131$, A allele: $\mathrm{p}=0.042$ ) (Table 3). Similarly, the A allele seems to be a factor affecting the age at onset of PD (Table 3), as carrying the allele associates with late onset ( $\geq 60$ years; $54.7 \%$ vs $45.3 \%$ ), and non-carrying associates with earlier onset ( $<60$ years; $67.9 \%$ vs $32.1 \%)(\mathrm{OR}=2.546,95 \% \mathrm{CI}=1.020-6.351, \mathrm{p}=0.042$ for $\mathrm{AA}+\mathrm{AG}$ vs. GG) (Figure $2 \mathrm{~B})$.

Three investigated SNPs of the IDO1 gene were not significantly correlated with PD patients, compared to healthy controls. However, the age-dependent subgroup analysis revealed that rs7820268 SNP or rs9657182 SNP carriers onset PD later in life compared to noncarriers, suggesting the rs7820268 or rs9657182 SNPs potentially delyed the disease onset of PD.

\section{Discussion}

DA receptors, catechol-O-methyltransferase, monoamine oxidase B, NMDA receptors, adenosine A2A receptors, and cholinergic receptors are main targets of PD medication approved for clinical use. The drugs frequently cause serious side effects, become less effectual during treatment, and eventually lead to development of drug-resistant PD such as L-DOPA-resistant PD [31,32]. Thus, a search for novel therapeutic targets is under extensive research. Intervention in KP of TRP metabolism has been shed light on in search of novel drugs for neurodegenerative and psychiatric diseases. Glutamate and acetylcholine are main neurotransmitters responsible for cognition and behavior in which bioactive KYNs mediate neurotoxic, neuromodulatory, and immunological response in reaction to pathological insults [3,33].

The decreased concentrations of L-KYN and KYNA in the frontal cortex, putamen and SNpc, and elevated concentrations of 3-HK in the putamen and SNpc were observed in postmortem brain of PD patients [34]. In addition to the central nervous system, peripheral samples showed 
abnormalities such as the increased activity of kynurenine aminotransferase (KAT) and elevated KYNA levels in red blood cells, which may be a protective response [35]. Natural product curcumin was reported to relieve pain and stress through the KYN metabolic pathway [36]. The KP is proposed to be a potential target for treatment of Alzheimer's diseases and schizophrenia [26,37]. Psychedelic psilocybin was reported to relieve depressive and anxiety symptoms of patients with terminal illness [38]. The action is considered at least partly through the KP. Furthermore, delivery of active agents to the brain through the blood-brain barrier is under extensive research [39]. Other in vitro and in vivo studies showed therapeutic opportunities through elevated levels of KYNA in PD [40-42].

However, no study reported a role of the gene polymorphism of KYN enzymes in PD pathogenesis. SNP variants of KMO were investigated. SNP variants of enzymes of the KP in PD were studied previously. SNP analysis of KMO in PD and healthy controls were compared and a subgroup analysis was conducted according to age of onset. Four loci of SNPs did not reveal any significance between PD and healthy controls and between subgroups of age of onset [43]. This study revealed that three SNPs of IDO1 were not associated with PD, but the rs7820268 and the rs9657182 SNPs affected the age of onset of PD. The A alleles carriers were associated with late age of onset, whereas non-carries were associated with early age of onset of PD. The A allele of rs7820268 SNP associated with later onset, whereas non-carriers of the allele were associated with earlier onset (Figure 2A). Another DNA strand the T allele of the SNP was shown to impair CD8+ Treg function [29]. The allele may lead to beneficial immunosuppressive effects in the rs7820268 SNP carriers in PD. The rs9657182 SNP is linked to moderate or severe IFN- $\alpha$-induced depressive symptoms in Caucasian patients with chronic hepatitis C. The SNP is located in the linkage disequilibrium block encompassing 20 kilobases upstream of IDO1 as well as includes at least six additional polymorphisms that may contain binding sites for immunoregulatory proteins or may have epigenetic regulatory function [30]. In addition, a downstream metabolite kynurenic acid was found an antidepressant in animal model of depression [44]. Indoximod is a methylated TRP that inhibits IDO to maintain or elevate TRP levels important to T cell function. Interestingly, a case study reported that indoximod induced parkinsonism in a patient treated for metastatic breast cancer.

Age is the most important risk factors for neurodegenerative diseases. Age-related risk of leptin metabolism disturbance, especially in obese people was associated with partial pathomechanism of neurodegenerative diseases [45]. Beneficial use of nutraceutical inositols was proposed for age-prone disturbances of energy metabolism and mitochondrial function [46]. In addition, genetic, environmental, infectious, nutritional, and lifestyle are initiation factors which play a role of pathogenesis of PD. Mutations in the genes SNCA, PRKN, LRRK2, PINK1, DJ-1, VPS35 and GBA have been shown to be important risk factors for PD [47]. The frequency of the L444P mutation of the GBA gene was found higher in the PD patients and associated with the early onset of PD [48].

Long interspersed element-1 (LINE-1) is a class I transposable element that has been linked to the various diseases including PD. Higher germline variations and increasing somatic variations of LINE-1 loci were associated with PD. Thus, polymorphism and accumulation of retrotransposition competent LINE-1s were considered to contribute to pathogenesis of PD [49]. The brain cytoplasmic 200 long-noncoding RNA 1 is coded in the BCYRN1. The rs13388259 Intergenic polymorphism $B C Y R N 1$ gene was associated with PD [50]. A large-scale meta-analysis of genome-side association (GSA) studies identified SNP variants of several loci associated with PD. GBA, GAK/DGKQ, SNCA, and $H L A$ were identified as secondary independent risk factors. Furthermore, substantial cumulative risk was reported by risk profile analysis [51]. GSA generated polygenic risk scores (PRS) for prediction of risk and progression of Parkinson's disease (PD), to search PD-related biological pathways from large-scale pathway specific-genetic risk profiling, and to identify causal PD genes and potential therapeutic targets [52]. Furthermore, increasing evidence suggests that epigenetic mechanisms play a role in regulation of PD-associated genes, including DNA methylation, histone modifications, and microRNAs (miRNSs) [53]. miRNAs are short non-coding RNAs responsible for gene expression at the post-transcriptional level. Altered miRNAs have been linked to mitochondrial dysfunction and oxidative stress in ageing and neurodegenerative diseases [54]. miR-133b, miR-7, miR153, miR-433, miR-433, miR-205, and miR-124 are of interest as possible biomarkers in PD [55]. 
Furthermore, differential monitoring of the redox status was proposed to build a personalized treatment for patients with multifactorial neurologic diseases [56]

This study had several limitations including its relatively small sample size, the small number of SNP loci, heterogeneity of the study subjects, and the collection of blood samples at a single center. Furthermore, enzyme activities of IDO1, IDO2, and TDO were not measured. Thus, it was not known if IDO2 and/or TDO compensated the polymorphic variants of IDO1. Prospective studies in larger patient number, with the larger number of SNP loci, and data with IDO2 and TDO activities are needed to validate our findings.

\section{Conclusion}

This study explored the potential role of IDO1 gene polymorphisms in PD. None of three IDO1 SNPs investigated in this study were significantly associated with PD; however, two IDO1 SNPs showed correlated with the age onset of $\mathrm{PD}$, suggesting that the gene polymorphisms may not play a direct role in pathogenesis of PD, but may influence the disease onset probably as secondary risk factors. The further investigation is expected in search of roles of gene polymorphism in risk, onset, prognosis, progression of PD including SNPs, structural variants, and the disease-related pathways.

Author Contributions: Conceptualization, N.T. and L.V.; methodology, N.T. and K.M.; software, N.T.; validation, N.T.; formal analysis, N.T. and K.M.; investigation, N.T.; resources, N.T., R. M.T., Z.S and F.S.; data curation, N.T. and K.B.; writing-original draft preparation, N.T.; writing-review and editing, M.T.; visualization, N.T.; supervision, P.K. and L.V.; project administration, N.T.; funding acquisition, L.V. All authors have read and agreed to the published version of the manuscript.

Funding: The current work was supported by the current work was supported by the Economic Development and Innovation Operational Programme (GINOP) GINOP 2.3.2-15-2016-00034, GINOP 2.3.2-15-2016-00048, TUDFO/47138-1/2019-ITM, and TKP2020 Thematic Excellence Programme 2020., University of Szeged Open Access Fund (5347).

Acknowledgments: The authors are sincerely grateful to Dr. Michael D. Lovelace and his co-workers for critical reading and their kind help.

Conflicts of Interest: The authors declare no conflicts of interest.

Acknowledgments: We want to thank Emilija Knezevic for technical support and making figures.

\section{Abbreviations}

$\begin{array}{ll}\text { AMPA } & \alpha \text {-amino-3-hydroxy-5-methyl-4-isoxazolepropionic acid } \\ \text { CSF } & \text { cerebrospinal fluid } \\ \text { DA } & \text { dopamine } \\ \text { 3-HAA } & \text { 3-hydroxyanthranillic acid } \\ \text { 3-HK } & \text { 3-hydroxykynurenine } \\ \text { IDO } & \text { indoleamine 2,3-dioxygenase } \\ \text { IFN } & \text { Interferon } \\ \text { KAT } & \text { kynurenine aminotransferases } \\ \text { KMO } & \text { kynurenine 3-monooxygenase } \\ \text { KP } & \text { kynurenine pathway } \\ \text { KYNA } & \text { kynurenic acid } \\ \text { NMDA } & \text { N-methyl-D-aspartate } \\ \text { PD } & \text { Parkinson's disease } \\ \text { QUIN } & \text { quinolinic acid }\end{array}$




$\begin{array}{ll}\text { ROS } & \text { reactive oxygen species } \\ \text { SNP } & \text { single nucleotide polymorphisms } \\ \text { TDO } & \text { tryptopan 2,3-dioxygenase } \\ \text { TRP } & \text { tryptophan }\end{array}$

\section{References}

1. Wu, T.; Hallett, M.; Chan, P. Motor automaticity in Parkinson's disease." Neurobiol. Dis. 2015, 82, 226234.

2. Dickson, D.W.; Fujishiro, H.; Orr, C.; DelleDonne, A.; Josephs, K.A.; Frigerio, R.; Burnett, M.; Parisi, J.E.; Klos, K.J.; Ahlskog, J.E. Neuropathology of non-motor features of Parkinson disease. Parkinsonism Relat. Disord. 2009, 15 Suppl 3:S1-5.

3. Tanaka, M.; Bohár, Z.; Vécsei, L. Are Kynurenines Accomplices or Principal Villains in Dementia? Maintenance of Kynurenine Metabolism. Molecules 2020, 25, 564.

4. Pringsheim, T.; N. Jette, A.; Frolkis, A.; Steeves, T.D. The prevalence of Parkinson's disease: a systematic review and meta-analysis. Mov. Disord. 2014, 29(13), 1583-1590.

5. Caudle, W.M.; Zhang, J. Glutamate, excitotoxicity, and programmed cell death in Parkinson disease. Exp. Neurol. 2009, 220(2), 230-233.

6. Reale, M.; Iarlori, C.; Thomas, A.; Gambi, D.; Perfetti, B.; Di Nicola, M.; Onofrj, M. Peripheral cytokines profile in Parkinson's disease. Brain Behav. Immun. 2009, 23(1), 55-63.

7. Blandini, F. An update on the potential role of excitotoxicity in the pathogenesis of Parkinson's disease. Funct. Neurol. 2010, 25(2), 65-71.

8. Török, N.; Majláth, Z.; Szalárdy, L.; Vécsei L. Investigational $\alpha$-synuclein aggregation inhibitors: hope for Parkinson's disease. Expert Opin. Investig. Drugs 2016, 25(11), 1281-1294.

9. Spatola, M.; Wider C. Genetics of Parkinson's disease: the yield. Parkinsonism Relat. Disord. 2014, 20 Suppl 1, S35-38.

10. Tanaka, M.; Toldi, J.; Vécsei, L. Exploring the Etiological Links behind Neurodegenerative Diseases: Inflammatory Cytokines and Bioactive Kynurenines. Int. J. Mol. Sci. 2020, 21, 2431.

11. Török, N.; Majláth, Z.; Fülöp, F.; Toldi, J.; Vécsei, L. Brain Aging and Disorders of the Central Nervous System: Kynurenines and Drug Metabolism. Curr. Drug Metab. 2016, 17(5), 412-429.

12. Hunt, C.; Macedo, E.; Cordeiro, T.; Suchting, R.; de Dios, C.; Leal, C.V.A.; Soares, J.C.; Dantzer R, Teixeira, A.L.; Selvaraj, S. Effect of immune activation on the kynurenine pathway and depression symptoms - A systematic review and meta-analysis. Neurosci. Biobehav. Rev. 2020, 118, 514-523.

13. Cao, B.; Chen, Y.; Ren, Z.; Pan, Z.; McIntyre, R.S.; Wang, D. Dysfunction of Kynurenine Pathway and Potential Dynamic Changes of Kynurenine in Schizophrenia: A Systematic Review and Meta-Analysis. Submitted to Neurosci. Biobehav. Rev. 2020.

14. Jovanovic, F.; Candido, K.D.; Knezevic, N.N. The Role of the Kynurenine Signaling Pathway in Different Chronic Pain Conditions and Potential Use of Therapeutic Agents. Int. J. Mol. Sci. 2020, 21, 6045.

15. Carrillo-Mora, P.; Pérez-De la Cruz, V.; Estrada-Cortés, B.; Toussaint-González, P.; Martínez-Cortéz, J.A.; Rodríguez-Barragán, M.; Quinzaños-Fresnedo, J.; Rangel-Caballero, F.; Gamboa-Coria, G.; Sánchez-Vázquez, I.; Barajas-Martínez, K.; Franyutti-Prado, K.; Sánchez-Chapul, L.; Ramírez-Ortega, D.; Ramos-Chávez, L.A. Serum Kynurenines Correlate With Depressive Symptoms and Disability in Poststroke Patients: A Cross-sectional Study. Neurorehabilit. Neural Repair 2020, 154596832095367.

16. Hunt, C.; Macedo e Cordeiro, T.; Suchting, R.; de Dios, C.; Cuellar Leal, V.A.; Soares, J.C.; Dantzer, R.; Teixeira, A.L.; Selvaraj, S. Effect of immune activation on the kynurenine pathway and depression symptoms - A systematic review and meta-analysis. Neurosci. Biobehav. Rev. 2020, 118, 514.

17. Widner, B.; Leblhuber, F.; Fuchs, D. Increased neopterin production and tryptophan degradation in advanced Parkinson's disease. J. Neural. Transm. 2002, 109(2), 181-189.

18. Romero, R.; Chaemsaithong, P.; Yeo, L.; Docheva, N.; Chaiyasit, N. Fetal and Maternal Responses to Intraamniotic Infection. In Fetal and Neonatal Physiology, 5th ed.; Barichello, T., Ed.; Elsevier: Amsterdam, The Netherlands, 2017; Volume 1, pp. 144-159. 
19. Majláth, Z.; Török, N.; Toldi, J.; Vécsei, L. Memantine and Kynurenic Acid: Current Neuropharmacological Aspects. Curr. Neuropharmacol. 2016, 14(2), 200-209.

20. Schwarcz, R.; Bruno, J.P.; Muchowski, P.J.; Wu H.Q. Kynurenines in the mammalian brain: when physiology meets pathology. Nat. Rev. Neurosci. 2012 13(7), 465-477.

21. Tuboly, G.; Tar, L.; Bohar, Z.; Safrany-Fark, A.; Petrovszki, Z.; Kekesi, G.; Vécsei, L.; Pardutz A.: Horvath $\mathrm{G}$. The inimitable kynurenic acid: the roles of different ionotropic receptors in the action of kynurenic acid at a spinal level. Brain Res. Bull. 2015, 112, 52-60.

22. Prescott, C.; Weeks, A.M.; Staley, K.J.; Partin, K.M. Kynurenic acid has a dual action on AMPA receptor responses. Neurosci. Lett. 2006, 402, 108-112.

23. Rózsa, E.; Robotka, H.; Vécsei, L.; Toldi, J. The Janus-face kynurenic acid. J. Neural. Transm. 2008, 115, 1087-1091.

24. Stone, T.W. Does kynurenic acid act on nicotinic receptors? An assessment of the evidence. J. Neurochem. 2020, 152, 627-649.

25. Lugo-Huitron, R; Blanco-Ayala, T.; Ugalde-Muniz, P.; Carrillo-Mora, P.; Pedraza-Chaverri, J.; SilvaAdaya, D.; Maldonado, P.D.; Torres, I.; Pinzon, E.; Ortiz-Islas, E.; Lopez T.; Garcia, E.; Pineda, B.; TorresRamos, M.; Santamaria A.; La Cruz, V.P. On the antioxidant properties of kynurenic acid: free radical scavenging activity and inhibition of oxidative stress. Neurotoxicol. Teratol. 2011, 33(5), 538-547.

26. Koola, M.M. Galantamine-Memantine combination in the treatment of Alzheimer's disease and beyond. Psychiatry Res. 2020, 293, 113409.

27. Tomaszewska, E.; Muszyński, S.; Kuc, D.; Dobrowolski, P.; Lamorski, K.; Smolińska, K.; Donaldson, J.; Świetlicka, I.; Mielnik-Błaszczak, M.; Paluszkiewicz, P.; Parada-Turska, J. Chronic dietary supplementation with kynurenic acid, a neuroactive metabolite of tryptophan, decreased body weight without negative influence on densitometry and mandibular bone biomechanical endurance in young rats. PLoS One 2019, 14(12), e0226205.

28. Mandi, Y.; Vécsei, L. "The kynurenine system and immunoregulation." J. Neural. Transm. 2012, 119(2), 197-209.

29. Tardito, S.; Negrini, S.; Conteduca, G.; Ferrera, F.; Parodi, A.; Battaglia, F.; Kalli, F.; Fenoglio, D.; Cutolo M.; Filaci G. "Indoleamine 2,3 dioxygenase gene polymorphisms correlate with CD8+ Treg impairment in systemic sclerosis. Hum. Immunol. 2013, 74(2), 166-169.

30. Smith, A.K.; Simon, J.S.; Gustafson, E.L.; Noviello, S.; Cubells, J.F.; Epstein, M.P.; Devlin, D.J.; Qiu, P.; Albrecht, J.K.; Brass, C.A.; Sulkowski, M.S.; McHutchinson J.G.; Miller, A.H. Association of a polymorphism in the indoleamine- 2,3-dioxygenase gene and interferon-alpha-induced depression in patients with chronic hepatitis C. Mol. Psychiatry 2012, 17(8), 781-789.

31. Dezsi, L.; Vécsei; L. Clinical implications of irregular ADMET properties with levodopa and other antiparkinson's drugs." Expert Opin. Drug Metab. Toxicol. 2014, 10(3), 409-424.

32. Calabresi, P.; Ghiglieri, V.; Mazzocchetti, P.; Corbelli I.; Picconi, B. Levodopa-induced plasticity: a double-edged sword in Parkinson's disease? Philos. Trans. R. Soc. Lond. B. Biol. Sci. 2015, 370(1672).

33. Stone, T.W.; Darlington L.G. The kynurenine pathway as a therapeutic target in cognitive and neurodegenerative disorders. Br. J. Pharmacol. 2013, 169(6), 1211-1227.

34. Ogawa, T.; Matson, W.R.; Beal, M.F.; Myers, R.H.; Bird, E.D.; Milbury, P.; Saso, S. Kynurenine pathway abnormalities in Parkinson's disease. Neurology 1992, 42(9), 1702-1706.

35. Hartai, Z.; Klivenyi, P.; Janaky, T.; Penke, B.; Dux, L.; Vécsei, L. Kynurenine metabolism in plasma and in red blood cells in Parkinson's disease. J. Neurol. Sci. 2005, 239(1), 31-35.

36. Zhang, Y.; Li, L.; Zhang, J. Curcumin in antidepressant treatments: An overview of potential mechanisms, pre-clinical/clinical trials and ongoing challenges. Basic Clin. Pharmacol. Toxicol. 2020 Jun 16.

37. Koola, M.M.; Jafarnejad, S.; Looney, S.; Praharaj, S.; Pillai, A.; Ahmed, A.; Slifstein, M. Meta-Analyses of Randomized Controlled Trials and Potential Novel Combination Treatments in Schizophrenia. Biol. Psychiatry 2020, 87(9), S306.

38. Vargas, A.S.; Luís, Â.; Barroso, M.; Gallardo, E.; Pereira, L. Psilocybin as a New Approach to Treat Depression and Anxiety in the Context of Life-Threatening Diseases-A Systematic Review and MetaAnalysis of Clinical Trials. Biomedicines 2020, 8, 331.

39. Rog, J.; Błażewicz, A.; Juchnowicz, D.; Ludwiczuk, A.; Stelmach, E.; Kozioł, M.; Karakula, M.; Niziński, P.; Karakula-Juchnowicz, H. The Role of GPR120 Receptor in Essential Fatty Acids Metabolism in Schizophrenia. Biomedicines 2020, 8, 243. 
40. Lee, D.Y.; Lee, K.S.; Lee, H.J, Noh, Y.H.; Kim, D.H; Lee, J.Y.; Cho, S.H.; Yoon, O.J.; Lee, W.B.; Kim, K.Y.; Chung, Y.H.; Kim, S.S. Kynurenic acid attenuates MPP(+)-induced dopaminergic neuronal cell death via a Bax-mediated mitochondrial pathway. Eur. J. Cell Biol. 2008, 87(6), 389-397.

41. Miranda, A.F.; Boegman, R.J.; Beninger, R.J.; Jhamandas, K. Protection against quinolinic acid-mediated excitotoxicity in nigrostriatal dopaminergic neurons by endogenous kynurenic acid. Neuroscience 1997, 78(4), 967-975.

42. Butler, E.G., Bourke, D.W. Finkelstein, D.I.; Horne, M.K. The effects of reversible inactivation of the subthalamo-pallidal pathway on the behaviour of naive and hemiparkinsonian monkeys." J. Clin. Neurosci. 1997, 4(2), 218-227.

43. Török, N.; Török, R.; Szolnoki, Z.; Somogyvári, F.; Klivényi, P.; Vécsei, L. The Genetic Link between Parkinson's Disease and the Kynurenine Pathway Is Still Missing. Parkinsons Dis. 2015, 2015, 474135.

44. Tanaka, M..; Bohár, Z.; Martos, D.; Vécsei, L. Antidepressant-like effects of kynurenic acid in a modified forced swim test. Pharmacol. Rep. 2020, 72, 449-455.

45. Pérez-Pérez, A.; Sánchez-Jiménez, F.; Vilariño-García, T.; Sánchez-Margalet, V. Role of Leptin in Inflammation and Vice Versa. Int. J. Mol. Sci. 2020, 21, 5887.

46. López-Gambero, A.J.; Sanjuan, C.; Serrano-Castro, P.J.; Suárez, J.; Rodríguez de Fonseca, F. The Biomedical Uses of Inositols: A Nutraceutical Approach to Metabolic Dysfunction in Aging and Neurodegenerative Diseases. Biomedicines 2020, 8, 295.

47. Aasly, J.O. Long-Term Outcomes of Genetic Parkinson's Disease. J. Mov. Disord. 2020, 13(2), 81-96.

48. Török, R.; Zádori, D.; Török, N.; Csility, É.; Vécsei, L.; Klivényi, P. An assessment of the frequency of mutations in the GBA and VPS35 genes in Hungarian patients with sporadic Parkinson's disease. Neurosci. Lett. 2016, 610, 135-138.

49. Pfaff, A.L.; Bubb, V.J.; Quinn, J.P.; Koks, S. An increased burden of highly active 3 retrotransposition competent L1s is associated with 4 Parkinson's disease risk and progression in the PPMI 5 cohort. Int. J. Mol. Sci. 2020 (submitted).

50. Márki, S.; Göblös, A.; Szlávicz, E.; Török, N.; Balicza, P.; Bereznai, B.; Takáts, A.; Engelhardt, J.; Klivényi, P.; Vécsei, L.; Molnár, M.J.; Nagy, N.; Széll, M. The rs13388259 Intergenic Polymorphism in the Genomic Context of the BCYRN1 Gene Is Associated with Parkinson's Disease in the Hungarian Population. Parkinsons Dis. 2018, 2018, 9351598.

51. Nalls, M.A.; Pankratz, N.; Lill, C.M.; Do, C.B.; Hernandez, D.G.; Saad, M.; DeStefano, A.L.; Kara, E.; Bras, J.; Sharma, M.; Schulte, C.; Keller, M.F.; Arepalli, S.; Letson, C.; Edsall, C.; Stefansson, H.; Liu, X.; Pliner, H.; Lee, J.H.; Cheng, R.; Ikram, M.A.; Ioannidis, J.P.; Hadjigeorgiou, G.M.; Bis, J.C.; Martinez, M.; Perlmutter, J.S.; Goate, A.; Marder, K.; Fiske, B.; Sutherland, M.; Xiromerisiou, G.; Myers, R.H.: Clark, L.N.; Stefansson, K.; Hardy, J.A.; Heutink, P.; Chen, H.; Wood, N.W.; Houlden, H.; Payami, H.; Brice, A.; Scott, W.K.; Gasser, T.; Bertram, L.; Eriksson, N.; Foroud, T.; Singleton, A.B. "Large-scale metaanalysis of genome-wide association data identifies six new risk loci for Parkinson's disease." Nat. Genet, 2014, 46(9), 989-993.

52. Hall, A.; Bandres-Ciga, S.; Diez-Fairen, M.; Quinn, J.P.; Billingsley, K.J. Genetic Risk Profiling in Parkinson's Disease and Utilizing Genetics to Gain Insight into Disease-Related Biological Pathways. Int. J. Mol. Sci. 2020, 21, 7332.

53. Navarro-Sánchez, L.; Águeda-Gómez, B.; Aparicio, S.; Pérez-Tur, J. Epigenetic Study in Parkinson's Disease: A Pilot Analysis of DNA Methylation in Candidate Genes in Brain. Cells 2018, 7, 150.

54. Brito, L.M.; Ribeiro-dos-Santos, Â.; Vidal, A.F.; de Araújo, G.S., on behalf of the Alzheimer's Disease Neuroimaging Initiative; Differential Expression and miRNA-Gene Interactions in Early and Late Mild Cognitive Impairment. Biology 2020, 9, 251.

55. Catanesi, M.; d'Angelo, M.; Tupone, M.G.; Benedetti, E.; Giordano, A.; Castelli, V.; Cimini, A. MicroRNAs Dysregulation and Mitochondrial Dysfunction in Neurodegenerative Diseases. Int. J. Mol. Sci. 2020, 21, 5986.

56. Tanaka, M.; Vécsei, L. Monitoring the Redox Status in Multiple Sclerosis. Preprints 2020, 2020070737(d).

57. Miller, S. A.; Dykes D.D.; Polesky, H.F. A simple salting out procedure for extracting DNA from human nucleated cells. Nucleic Acids Res. 1988, 16(3), 1215. 